\title{
Discursos sobre la evaluación del desempeño docente en el contexto de la reforma educativa de 2013 en México
}

\author{
Discourses on teacher performance assessment in the context of the educational reform of 2013 in
}

Mexico

\section{Haideé Franco Moreno ${ }^{a}$}

\begin{abstract}
:
The purpose of this paper is to analyze the discourse production dictated by international organizations to justify the application of teachers performance evaluation in Mexico, as the key device to ensure educational quality in the National Educational System (SEN by its Spanish initials) with the intent to discuss the main arguments that during the previous federal government administration worked as the basis of defining their public policy addressed to elementary and high school education within the context of the Education Reform started in 2013. It is concluded that the main discourse reveals the necessity of measuring teachers' teaching skills and aptitudes in order to guarantee the quality in education; it strengthens the teaching profession through defining teaching performance criteria; it links the concepts of suitability with the one of teachers' performance levels and finally, it holds teachers responsible for students learning results.
\end{abstract}

\section{Keywords:}

Discourse Analysis, Teachers Performance Evaluation, Education Reform, International Organisms

\section{Resumen:}

El objetivo de este trabajo es analizar la producción de los discursos dictados por los organismos internacionales para justificar la implementación de la evaluación del desempeño docente en México, como el dispositivo clave para asegurar la calidad educativa en el Sistema Educativo Nacional (SEN), con la intención de discutir los principales argumentos que en el sexenio anterior funcionaron como punto de partida en la definición de política pública dirigida al nivel de educación básica y media superior en el marco de la reforma educativa iniciada en el año 2013. Se concluye que los principales discursos revelan la necesidad de medir las capacidades y aptitudes de los profesores para garantizar la calidad de la educación; buscan fortalecer la profesión docente mediante la definición de criterios de desempeño; vinculan el concepto de idoneidad con niveles de desempeño de los profesores y finalmente, responsabilizan a los docentes de los resultados de aprendizaje de sus alumnos.

\section{Palabras Clave:}

Análisis del Discurso, Evaluación del Desempeño Docente; Reforma Educativa; Organismos Internacionales

Ensayo recibido: 12 de septiembre de 2019

Dictaminado: 10 de octubre de 2019

Segunda versión: 16 de octubre de 2019

Aceptado: 21 de octubre de 2019 


\section{Introducción}

Para lograr una lectura de la realidad social es necesario realizar una interpretación de los discursos y los fines que éstos promueven en el mundo a través de los enunciados que cotidianamente circulan, en donde las palabras y los signos son los medios para expresar los pensamientos y sirven para la reconstrucción del contexto. Este paradigma le reconoce al lenguaje una función no sólo referencial (informativa) y epistémica (interpretativa), sino también creativa (Echeverría 2003). Por lo tanto, en este trabajo se busca dar cuenta, desde esas tres funciones del discurso (referencial, epistémica y creativa), sobre la forma en que influyen los organismos internacionales en el diseño de política pública de distintos países, ya que éstos se han erigido como los principales responsables para evaluar los sistemas educativos nacionales y redactar los informes con recomendaciones y sugerencias de lo que puede mantenerse y lo que habría que desecharse.

México vivió un proceso de reforma educativa que inició en 2013 y que concluyó justamente en este año (2019); uno de los propósitos de dicha reforma fue la búsqueda de mejores resultados en el logro escolar, a partir de asegurar la calidad profesional de los docentes de los niveles de educación básica y media superior. Desde entonces, la atención se ha centrado en el rostro más visible de esa reforma educativa que es la evaluación del desempeño docente. El argumento central para evaluar a los profesores fue que "los docentes son el factor clave para asegurar la calidad de la educación que reciben los estudiantes" (INEE, 2015: 11). Sin embargo, aunque el enfoque sobre los derechos humanos se mostraba explícito en el discurso, cuando se expresó que es un derecho humano de todos tener una educación de calidad (INEE, 2015: 9), en el documento Los docentes en México. Informe 2015, no se mencionan los mecanismos para hacer realidad el ejercicio de ese derecho, ya que las desigualdades aún persisten, como el rezago educativo y la falta de cobertura en el Sistema Educativo Nacional (SEN). Asimismo, en dicho documento se apela al interés superior de la niñez, pero en su momento, no se garantizaron los derechos laborales del magisterio mexicano, al aprobar las leyes secundarias contenidas en la Ley General del Servicio Profesional Docente (LGSPD).

Por otro lado, en el Acuerdo de cooperación MéxicoOCDE para mejorar la calidad de la educación de las escuelas mexicanas se mencionó que "la reforma más importante en política pública que puede hacer México para mejorar los resultados educativos de sus jóvenes es construir un sistema sólido que permita seleccionar, preparar, desarrollar y evaluar a los mejores docentes para sus escuelas" (OCDE, 2010). De este enunciado, puede observarse que las decisiones en materia educativa han sido dictadas por organismos internacionales como la Organización para la Cooperación y el Desarrollo Económicos (OCDE), el Banco Mundial (BM) y el Banco Interamericano de Desarrollo (BID). Puede por tanto, afirmarse que los organismos internacionales buscan asegurar la calidad educativa a través del establecimiento de estándares, lo cual perfila una organización racional del trabajo docente que busca maximizar su eficiencia laboral a través de la necesaria estandarización y homogeneización de todos los procesos escolares, reduciendo las formas de ejercer la docencia y la gestión directiva a una forma preestablecida de proceder, a lo prescrito y a la eliminación de imprevistos, asumiendo con ello una racionalidad neoliberal en la producción del discurso.

Para lograr la institucionalización de la evaluación de profesores, se firmó el 2 de diciembre de 2012, el Pacto por México, suscrito por el entonces Presidente Enrique Peña Nieto y los líderes de las principales fuerzas políticas (PRI, PAN y PRD') estableciéndose modificaciones en el artículo $3^{\circ}$ Constitucional y se generaron dos leyes secundarias: la Ley del Instituto Nacional para la Evaluación de la Educación (LINEE) y la Ley General del Servicio Profesional Docente (LGSPD). Fue entonces que el discurso sobre la calidad educativa en México fue construido a partir de diversos lineamientos establecidos por los organismos internacionales, quienes aparecieron al plantear una 
serie de recomendaciones con criterios a seguir, mismos que fueron plasmados en numerosos documentos e informes que permearon en la educación básica y media superior a nivel nacional, estatal y local. Es desde este contexto, que se analizarán los principales discursos producidos durante el sexenio 2012-2018, que promovieron la evaluación del desempeño de profesores en México, como el argumento principal para mejorar la calidad de la educación.

Es verdad que la necesidad de implementar un proceso para evaluar el desempeño de los profesores, como soporte de la reforma educativa implementada en el 2013, tuvo que ver con el requerimiento de conocer cuáles eran las áreas de mejora para emprender acciones de solución y en consecuencia garantizar la calidad educativa. Sin embargo, la forma en la que se establecieron los discursos para legitimar ese proceso siguió una lógica en donde la realidad educativa nacional no se tomó como punto de partida sino de llegada, en tanto que el diseño de la política educativa se planeó y se ejecutó con una orientación vertical "de arriba hacia abajo".

\section{Cuerpo argumentativo}

\section{1.- El discurso que señalaba la necesidad de medir las capacidades y aptitudes de los profesores para garantizar la calidad de la educación}

De acuerdo con Fardella (2012), las políticas educativas han tenido un giro significativo en las últimas dos décadas, asumiendo la racionalidad neoliberal, donde los cambios adoptados por los últimos gobiernos se han dirigido hacia el quehacer docente, instalando nuevas regulaciones laborales referidas al trabajo docente. El 8 de agosto de 2002, durante el gobierno del presidente Vicente Fox Quesada fue creado, por decreto presidencial, el Instituto Nacional para la Evaluación de la Educación (INEE). Antes de constituirse como órgano autónomo, operó, en una primera etapa (del 8 de agosto, 2002 al 15 de mayo, 2012), como un organismo descentralizado de la Secretaría de Educación Pública; en una segunda (del 16 de mayo, 2012 al 25 de febrero,
2013), como un organismo descentralizado no sectorizado (INEE, 2017).

Como se estableció en el decreto, publicado en el Diario Oficial de la Federación ([DOF], 2013), a partir del 26 de febrero de 2013, el Instituto Nacional para la Evaluación de la Educación se convirtió en un organismo público autónomo, con personalidad jurídica y patrimonio propio. En esta nueva etapa, el INEE tuvo como tarea principal evaluar la calidad, el desempeño y los resultados del Sistema Educativo Nacional en la educación preescolar, primaria, secundaria y media superior. Entre sus responsabilidades estaban diseñar y realizar las mediciones que correspondan a componentes, procesos o resultados del sistema; expedir los lineamientos a los que se sujetarán las autoridades educativas federal y locales para llevar a cabo las funciones de evaluación que les correspondan y, generar y difundir información para, con base en ésta, emitir directrices que fueran relevantes para contribuir en las decisiones tendientes a mejorar la calidad de la educación y su equidad como factor esencial en la búsqueda de la igualdad social (INEE, 2017).

Aunado a lo anterior, el INEE coordinaba el Sistema Nacional de Evaluación Educativa (SNEE) y tenía la responsabilidad de definir los métodos e instrumentos de evaluación destinados a medir las capacidades y aptitudes de quienes aspiraban a ingresar al magisterio, así como de quienes se proponían obtener el ascenso a cargos de dirección y de supervisión en los ámbitos de educación básica y media superior, de igual modo, para evaluar el desempeño del magisterio en servicio (INEE, 2017). Desde ese discurso se observa la creación de un modelo de evaluación de índole eficientista, es decir, que se requiere y perfila a un "docente técnico", convirtiéndolo en un especialista en técnicas y recursos para solucionar problemas, donde la evaluación del desempeño docente se configuraba como el argumento eje para implementar, con fuerza, un nuevo prototipo de profesionalidad docente: la enajenación del actuar docente mediante la reducción a formas preestablecidas de ejercer la enseñanza. 
Ante ese panorama, el INEE se planteó la necesidad de "contar con un diagnóstico sobre aspectos clave de los docentes en México para poder ofrecer datos confiables" (INEE, 2015:10). El Servicio Profesional Docente (SPD), a través de la Coordinación Nacional del Servicio Profesional Docente (CNSPD), estaría entendido como una herramienta de política integral de la educación obligatoria con procesos articulados orientados hacia la promoción de la calidad educativa que debería buscar, principalmente, el fortalecimiento de la profesión. Sin embargo, el enfoque epistémico de proceso-producto, para evaluar la efectividad de los profesores y obtener la mejora del rendimiento del actuar docente, genera la percepción de un escenario de mantención de competencias mínimas y escasas posibilidades de un desarrollo continuo y permanente.

Las evaluaciones del desempeño son consideradas como "oportunidades de mejora" de los profesores y tienen un alto impacto tanto positivo como negativo en su carrera docente (Cuenca, 2015: 29). En tanto que se introduce un mecanismo que afecta la permanencia en el servicio, este planteamiento rompe con la "estabilidad laboral y el valor de la experiencia traducida en años de servicio que aún caracteriza a la mayoría de las carreras docentes latinoamericanas" (Cuenca, 2015: 30). Por lo tanto, Fullan (2002) afirma que toda innovación debe ser considerada como parte de un universo de significados y los actores educativos no pueden ser ubicados como meros adoptantes. En este sentido, para Galaz (2015) la evaluación de los profesores debiera ser entendida como un proceso más complejo que la simple medición y constatación del cumplimiento de los estándares oficiales de desempeño, señala que diversos estudios sostienen que el proceso de ser evaluado puede tensionar el concepto que los profesionales construyen de Sí y de su trabajo, al restringir el campo de posicionamiento profesional de los profesores y al facilitar u obstaculizar la adhesión al sistema y al mejoramiento del desempeño.

En la regulación del Servicio Profesional Docente (SPD), se instituyó por primera vez en la historia del sistema educativo mexicano, un documento único, que integró todos los procesos de la vida profesional docente (ingreso, promoción, permanencia y reconocimiento), cuyos planteamientos ocuparon el nivel de una ley secundaria de la Constitución política mexicana, ésta fue la Ley General del Servicio Profesional Docente. En la cual, la evaluación del desempeño no sólo estaba asociada a los cuatro procesos del SPD, sino que era el eje central que los articulaba y organizaba a lo largo de la carrera docente, por lo tanto, todos estos procesos fueron una derivación del uso de los resultados de la evaluación del desempeño en sus diferentes niveles.

La evaluación del desempeño definía etapas, utilizaba distintos instrumentos y fuentes diversas (SEP, 2015) y cada nivel de desempeño integraba una serie de evidencias entregadas en varias etapas. Se hizo necesario que tanto el examen como las rúbricas utilizadas para evaluar los informes y argumentaciones solicitadas, si bien podían adaptarse y modificarse, deberían haber contemplado los perfiles, parámetros y criterios diferenciados en cada nivel para mantenerse coherentes con el modelo secuencial. Puede observarse que en este discurso la instalación del mérito es el principio rector para el ingreso y el desarrollo y la promoción en el servicio. La LGSPD consideraba, de carácter obligatorio, el proceso de permanencia y, de carácter voluntario, el proceso de promoción en la función; sin embargo, lo que era obligatorio era someterse a la evaluación del desempeño cuatrienalmente, y los resultados de la evaluación eran los que indicaban la permanencia, y promoción del examinado. Por tanto, el carácter voluntario de la promoción, en realidad no lo era tanto, ya que lo que se suponía voluntario era la solicitud de promoción en caso de obtener el máximo resultado en la evaluación, algo que, en principio, debería convenir a cualquier actor educativo que se encontrara en ese caso.

\section{2.- El discurso que buscaba fortalecer la profesión docente a través de criterios y estándares de desempeño}

Murillo, González y Rizo (2007) sostienen que desarrollar sistemas de evaluación del desempeño 
docente que contribuyan al desarrollo profesional de los maestros y con ello, a la mejora de la calidad de la enseñanza, es una de las principales preocupaciones de los sistemas educativos actuales. Por lo tanto, el establecimiento de un marco común para la buena enseñanza o criterios de desempeño profesional se entienden como la primera etapa que permite definir programas de formación de profesores y desarrollar sistemas de evaluación del desempeño profesional en los distintos sistemas educativos (OCDE, 2010a). De ese modo, el establecimiento de un Marco para la Buena Enseñanza (MBE) es un criterio internacional que sirve para orientar las políticas de fortalecimiento de la profesión docente pues contiene la base técnica para mejorar las propuestas para la formación de profesores (Manzi, 2015). Así la implementación de un MBE y el diseño de criterios de desempeño profesional para docentes son las principales tendencias para mejorar la calidad de la profesión a nivel internacional (Feldman y laies, 2010; Vaillant, 2004).

La definición de criterios de Buen Desempeño Docente o Marcos para la Buena Enseñanza es el primer peldaño en el proceso de construcción e implementación de políticas públicas dirigidas a fortalecer la evaluación del desempeño de los profesores en educación básica. Algunos sistemas educativos de América Latina han desarrollado importantes avances en sus propuestas de carrera docente, una de las principales tendencias para fortalecer la profesión docente ha sido la elaboración de marcos para la enseñanza o criterios de desempeño profesional. Entre las experiencias internacionales en el desarrollo de estándares de desempeño docente, destacan la de Estados Unidos, Australia, Chile, México, Perú y Colombia (Leyva, Serrato y Navarro, 2015). En el caso de Chile y México se establecieron niveles de desempeño que permiten identificar el grado de dominio de los componentes, descriptores y referentes, respectivamente. En ambos casos se propuso que la evaluación del desempeño docente fuera formativa; sin embargo, puede utilizarse como referente para realizar evaluaciones con fines sumativos y consecuencias de alto impacto (Vázquez, Cordero, y Leyva, 2014).
El caso chileno es el referente para su implementación en la región, por reconocer tanto la dimensión sociopolítica como la técnica y lograr uno los esquemas más conocidos y reconocidos de evaluación docente que se llevó a cabo después de un amplio proceso de discusión (Cordero y González, 2016). En Chile, prácticamente toda la formación continua está relacionada con el Marco para la Buena Enseñanza, en él se estructuran los dominios, criterios y descriptores (Alvarado y otros, 2011), la evaluación tiene propósitos sumativos y de alto impacto en la carrera docente, es decir, los resultados del proceso llegan a tener consecuencias administrativas, como el acceso a incentivos salariales o la obligación de abandonar la carrera docente cuando no se logran los estándares establecidos (Manzi, González y Sun, 2011).

Desde esa visión, la OCDE (2010b) señaló que la experiencia chilena podía aportar importantes elementos y aprendizajes útiles para la agenda de reformas educativas mexicana. Por su parte, la OREALC/UNESCO (2013) estableció que las políticas docentes deben ser abordadas desde una perspectiva integral y sistémica, por lo que es fundamental contar con una definición válida y consensuada de la buena enseñanza y estándares de desempeño docente.

Es necesario aclarar entonces que el término estándar es un concepto ampliamente discutido en el campo educativo por su origen en el sector industrial (Barrera y Myers, 2011). En educación, por un lado, se concibe como la definición de lo que debe saber y hacer un profesional en determinado contexto $y$, por otro, se define como un insumo para indicar la distancia entre el desempeño del evaluado y el nivel necesario para ser considerado competente (Meckes, 2014).

Se concibe pues, a la evaluación como un proceso individual, que reflejaría las competencias de un docente con independencia de las interacciones y condiciones en las que la profesión se configura en lo cotidiano, dicha acción tiene necesariamente una lógica neoliberal y conductista, pues denota el surgimiento de nuevas regulaciones laborales con indiscutibles consecuencias subjetivas, tales como la precarización laboral; la 
tecnificación de la práctica; la adaptación necesaria a un modelo de oferta-demanda y la preocupación sobre su permanencia en el sistema, donde "los docentes cuenten con una sólida preparación profesional con la cual enfrentar las exigencias del hecho educativo" (INEE: 2015:7). A pesar de que la evaluación sumativa no sea un objetivo propio del marco, los sistemas educativos utilizan estos criterios para realizar procesos de acreditación, promoción o incremento salarial de los docentes, no obstante, es importante valorar los riesgos que representa la evaluación de alto impacto para los fines formativos (Vázquez, Cordero, y Leyva, 2014).

Casassus (2010) menciona que la aplicación, durante 30 años, de mecanismos de medición de origen conductista no ha producido los resultados esperados. Es usual escuchar que la medición externa y objetiva de resultados referidos a estándares, es un instrumento eficaz para el logro de una educación de calidad, por lo que es sorprendente notar que a "pesar de la evidencia empírica de que estas políticas no han funcionado, la creencia en ellas se mantiene" (Casassus, 2010: 86). Por lo tanto, desde el punto de vista de la evaluación y el mejoramiento de la calidad de la educación, esas mediciones se han convertido en herramientas ineficaces y onerosas.

Como lo señala Casassus (2010: 89), "el punto a destacar es que el tema no es «inocente» la decisión de estandarizar es de naturaleza política y por lo tanto, sujeta al poder de decisión en el sentido de que para que un estándar pueda funcionar, debe existir un acuerdo necesario sobre lo que es calidad por parte de quienes están sometidos al estándar... en el caso de Chile, la decisión es tomada por la tecno/burocracia". Lo mismo ocurrió en México, donde diferentes sectores mediante foros, artículos y notas periodísticas cuestionaron el sentido, alcances e implicaciones de la evaluación del desempeño docente y su relación con la calidad educativa entre otros factores críticos; colectivos disidentes de profesores de educación básica manifestaron su desacuerdo con esta medida y realizaron movilizaciones sociales que intentaron abortar el proceso de reforma (Cordero y González, 2016).
Numerosos investigadores (Arnaut, 2015; Gil Antón, 2016; Flores-Crespo, 2016; Ornelas, 2016; Díaz-Barriga, 2016) han dado cuenta que la evaluación docente, así como estaba establecida en la LGSPD, continuaba asociada al control y la sanción más que al desarrollo profesional y a la mejora de las escuelas.

\section{3.- El discurso que vinculaba el concepto de idoneidad con el nivel de desempeño de los profesores}

El documento Los Docentes en México: Informe 2015 contiene "recomendaciones de política educativa para atender los problemas detectados y avanzar en el fortalecimiento de la profesión docente, precisamente en el sentido de reconocer a maestras y maestros como factor esencial del mejoramiento de la calidad y de la plena vigencia del derecho a la educación para todos" (INEE, 2015: 7). En ese mismo documento se establece que "es de la mayor relevancia para el país contar con información que permita conocer a los docentes en términos de quiénes son, dónde se forman profesionalmente, qué tan bien preparados están y bajo qué condiciones prestan sus servicios.

La reforma educativa del 2013 cimentó la carrera docente en la idoneidad y el mérito de los profesores; ese énfasis en el mérito puede entenderse como una derivación del concepto de calidad que se introdujo en la reforma del Artículo Tercero de la Constitución Mexicana. Si bien dicho concepto no fue definido en la Constitución ni se encontró su definición en las leyes secundarias, lo que sí quedó claramente establecido en esos documentos fueron los referentes para reconocer la idoneidad; éstos son: los Perfiles, Parámetros e Indicadores, que en su conjunto integran el Marco General de la Calidad de la Educación (Diario Oficial de la Federación [DOF], 2013).

El reconocimiento de idoneidad, entendido como mérito, se consideró el requisito indispensable para ingresar al servicio, mantenerse en él, promoverse u obtener algún tipo de reconocimiento, tales como horas adicionales, responsabilidades al interior de la escuela o estímulos salariales. Es así que el Estado buscó que los 
profesores tuvieran "de la carrera docente una profesión atractiva, no sólo por medio del mejoramiento salarial sino también mediante el diseño y la puesta en marcha de un sistema de incentivos que reconozca y premie el mérito" (INEE: 2015:7), ese modelo de incentivos permite reconocer tres grandes tipos de consecuencias: a) incentivos económicos, derivados del proceso de promoción en la función, b) recompensas profesionales, establecidas en el proceso de reconocimiento; y c) presión por rendir cuentas, expresada en el proceso de permanencia (Bruns y Luque, 2014).

Por primera vez en el sistema educativo mexicano, no se consideraron como criterios para la promoción vertical $u$ horizontal la antigüedad, los años de experiencia laboral, o la acumulación de constancias o certificaciones. Otra característica esencial fue el hecho de que el Estado se erigió como la única autoridad en la regulación de la carrera docente. Esta situación exigió que "los docentes contaran con una sólida preparación profesional con la cual enfrentar las exigencias del hecho educativo" (INEE: 2015:7). Puede observarse que la cuestión del mérito tiene un papel central para el Estado, quien se encarga de ser la instancia responsable de validar, verificar y controlar que los profesores alcancen los niveles de desempeño exigidos, pero no salvaguarda ni organiza instancias que fomenten el mantenimiento y la superación de habilidades y conocimientos profesionales mediante una formación en el servicio de los que no logran acreditar el estándar. Los principios pedagógicos, en ese argumento, son, el de una racionalidad técnica, cuya orientación permea en los distintos modelos latinoamericanos de evaluación que han aceptado las recomendaciones y sugerencias de los organismos internacionales.

La reforma educativa, que inició en México en el 2013, se consideró de gran calado, pues tenía como propósito elevar la calidad educativa del país e inició con la acción de someter a los profesores a una evaluación de su desempeño docente, mediante pruebas estandarizadas. El primer paso, como se mencionó, fue la promulgación de la LGSPD, que propuso estándares de evaluación únicos, denominados: Perfil, Parámetros e Indicadores de buena enseñanza (SEP, 2010). La Ley también contempla que estos estándares de buena práctica docente deberán pasar por procesos de validación y revisión periódica (DOF, 2013).

EI INEE estableció que el actuar docente "implica tomar decisiones, invertir recursos, hacer operar mecanismos cada vez más eficientes y legítimos" (INEE, 2015:12). Ciertamente, todas estas operaciones son altamente racionales y van configurando a la docencia como una actividad que pareciera tener un núcleo fundamental en el uso y manejo correcto de toda la información en la toma de decisiones, la cual constituye a la acción docente como un espacio táctico, eminentemente conductual, bajo los principios técnicos del accountability, tomando distancia de las subjetividades e intersubjetividades de los actores sociales y tomando un modelo que organiza la escuela con un enfoque taylorista, en pos del progreso y del desarrollo institucional a través de la planificación y la gestión de la técnica. Desde ese enfoque prescriptivo, a nivel del diseño, la evaluación del desempeño limita a los profesores al mero cumplimiento de estándares e instrucciones implementadas desde afuera.

“...dicha suposición no sólo es éticamente insostenible sino, también empíricamente falsa, pues precisamente lo que caracteriza la labor en la profesión docente es la imposibilidad de realizarla siguiendo pautas específicas y analíticamente prescritas" (Díaz-Barriga, 2010).

\section{4.- El discurso que señalaba a los profesores como los únicos responsables de los resultados de aprendizaje de los alumnos.}

Durante la implementación de la Reforma Educativa, se han realizado diversos cuestionamientos sobre la naturaleza de esta reforma, siendo calificada como: una reforma estrictamente laboral (Gil Antón, 2015), estructural (Ornelas, 2013; Zebadúa, 2013) o política (Rodríguez, 2015). Una de las críticas más fuertes gira sobre el hecho de que esta estrategia parece considerar a los profesores como los únicos responsables de los 
bajos resultados del sistema educativo mexicano en las evaluaciones internacionales (Gil Antón, 2016).

En América Latina, todos los países han desarrollado experiencias de evaluaciones nacionales centradas en el aprendizaje de los alumnos (Murillo y Román, 2010). Según el Informe McKinsey (Barber y Mourshed, 2007), la evidencia disponible sugiere que el principal impulsor de las variaciones en el aprendizaje escolar es la calidad de los docentes. Desde esta perspectiva, sería posible sostener que aquellos docentes evaluados con desempeño sobresaliente son los más efectivos en aula y obtienen mejores resultados de aprendizaje en los estudiantes, la calidad se reconoce en el logro de los aprendizajes, dado que "constituye una métrica clave de la calidad" (Banco Mundial, 2011: 4).

Actualmente, se da una gran importancia a los indicadores que obtienen los alumnos en las pruebas estandarizadas de gran escala en los diferentes informes que se presentan. Se cree así, que lograr puntajes más altos en estas pruebas, equivale a elevar la calidad educativa y el factor que se considera como principal para conseguirlo es el docente (MartínezChairez y otros, 2016).

La necesidad de evaluar a los profesores tendría que ver con la necesidad de conocer cuáles son las áreas de mejora para emprender acciones de solución, pero los resultados de las evaluaciones no se han usado, hasta el momento, para la toma de decisiones que incidan en la mejora del aprendizaje de los alumnos de educación básica (Navarro, 2017). Desde este argumento, la evaluación cuatrienal del desempeño docente con fines de permanencia en la función, parte del supuesto de que la valoración periódica del desempeño docente mejora los resultados del aprendizaje de los alumnos, pero tampoco se cuenta con evidencia suficiente para asegurar esta relación (Scott y otros, 2015).

En la investigación con enfoque mixto, realizada por Martínez-Chairez y otros (2016), no existe relación entre el desempeño docente y la calidad educativa; señalan, derivado de su investigación, que los maestros consideran como necesaria la evaluación, tanto de su propio desempeño como el de los alumnos. Sin embargo, establecen que se deben contemplar las condiciones particulares de cada alumno y docente: ubicación geográfica, condiciones materiales, tamaño de grupo, origen socioeconómico, entre otras. En otro estudio, de índole eminentemente cuantitativo, Vega y Galaz (2015) relacionan los factores que intervienen en el proceso de aprendizaje del alumno y el indicador de ser o no sometido a evaluación docente. Establecen que no habría mayor influencia del proceso de evaluación sobre los resultados de los estudiantes, por lo tanto, el sistema estaría respondiendo a la meta de cerciorarse que los profesores alcanzan los niveles deseados de desempeño, pero esta acción no garantiza una mejora en los aprendizajes de los alumnos.

A partir de estas investigaciones se advierte que la complejidad de la realidad educativa no puede simplificarse suponiendo que al evaluarse un profesor, con un resultado de idoneidad, se generarán en igual proporción resultados, mayor aprendizaje con sus alumnos. Sin embargo, éste ha sido el argumento eje para implementar con mayor fuerza un nuevo prototipo de profesionalidad docente que está buscando adaptarse a un modelo laboral eficientista y tecnocrático.

\section{Algunas reflexiones conclusivas para la apertura}

En todos los ámbitos de la vida humana y en la diversidad de los grupos sociales, la evaluación está presente y desempeña un papel fundamental, pero no todos los que se involucran en procesos de evaluación educativa lo hacen compartiendo las mismas concepciones sobre lo que es evaluación y sus finalidades, ni utilizan sus resultados de la misma manera; en otras palabras, no se comparte la misma cultura sobre la evaluación en educación (MorenoBayardo, 2016).

De acuerdo con Catalán y González (2009), la implementación de la evaluación docente implica un importante cambio que afecta las prácticas, creencias y sentimientos de los profesores, por lo que ellos se convierten en los principales actores de la implementación y, por lo tanto, resulta imprescindible 
tomarlos en cuenta a la hora de evaluar la viabilidad de cualquier propuesta. Se presupone que los docentes deberían de estar convencidos de entrada, de las bondades y ventajas de la reforma y su evaluación basada en estándares, sin planear que también podrían darse casos de oposición, resistencia o boicot en los procesos de evaluación. Por lo tanto, para Catalán y González (2009), se configura como imprescindible conocer la relación que existe entre la actitud de los profesores ante la evaluación del desempeño docente y la autoevaluación que hacen de su propio desempeño, señalando que es razonable pensar que los profesores puedan tener ciertas reticencias a ser evaluados, por las posibles consecuencias que les pudieran traer sus resultados.

Los mismos autores indican que en el proceso que se está llevando a cabo en Chile se han considerado aspectos técnicos y gremiales; pero aún falta incorporar aspectos propios de la subjetividad de los actores. Uno de ellos, es la posibilidad que los profesores emitan opiniones acerca del proceso de evaluación de su desempeño (Catalán y González, 2009). Por lo tanto, una manera de aproximarse a la forma como los profesores podrían enfrentar el proceso de evaluación de su desempeño es mediante el conocimiento de sus actitudes, entendidas como "disposiciones permanentes de ánimo formadas por un conjunto de convicciones y sentimientos que llevan al sujeto que las tiene a actuar y expresarse según ellas en sus actos y opiniones" (Sierra-Bravo, 1999: 371).

Siguiendo a Catalán y González (2009), se puede señalar que es pertinente y viable estudiar las actitudes de los profesores hacia su propia evaluación del desempeño e independientemente de estos resultados, sugieren desarrollar alguna intervención para mejorar la disposición de los profesores a involucrarse en el sistema de evaluación del desempeño docente. Establecen la necesidad de incrementar los esfuerzos en aspectos subjetivos e intersubjetivos de los sujetos de la evaluación, destacando que un cambio cultural no se produce abruptamente, sino que las futuras generaciones de profesores deberían apropiarse tempranamente de la cultura evaluativa. La importancia de entender la cultura de la profesión docente como el reflejo de un conjunto de creencias dominantes, valores, hábitos y normas que determinan lo que este grupo social considera en su profesión, incide en la conducta de rechazo activo a la evaluación. Por ejemplo, el temor a obtener un bajo resultado, además de percepciones negativas, como la falta de legitimidad de la evaluación en términos de sus criterios, instrumentos y obligatoriedad, así como la falta de información sobre el sistema de evaluación (Tornero y Taut, 2010).

Puede inferirse, a partir de los argumentos anteriores, que con la creación del INEE, el Estado instauró un aparato punitivo, en donde el proceso de evaluación del desempeño funcionó como un dispositivo de selección, sencillo pero individualizador, entre normales y anormales (Foucault, 1979). De manera análoga, los profesores fueron clasificados por el INEE como idóneos y no idóneos, ubicándolos en un determinado orden de clasificación para lograr individualización. Es decir, una sociedad disciplinaria que se logra por medio de "la distribución de los individuos en un determinado espacio" (Foucault, 1980: p. 154).

Desde esa explicación, puede observarse que el reconocimiento de idoneidad, entendido como mérito, se consideró en ese momento, el requisito indispensable para ingresar al servicio, mantenerse en él, promoverse u obtener algún tipo de reconocimiento, ya sean horas adicionales, responsabilidades al interior de la escuela 0 estímulos salariales. Por primera vez en el sistema educativo mexicano, no se consideraron como criterios para la promoción vertical u horizontal, la antigüedad, los años de experiencia laboral o la acumulación de constancias o certificaciones.

En el año 2015, los profesores considerados como "anormales", o bien, "no idóneos", fueron aquellos que decidieron no ser evaluados en el desempeño o aquellos que no aprobaron el proceso de evaluación del desempeño docente, éstos profesores fueron el ejemplo claro de las consecuencias asumidas en caso de no actuar según las reglas establecidas por la institución en la LGSPD. Partiendo de esta base, los procesos de 
subjetivación que se dan en la sociedad, particularmente a través de sus organizaciones, propenderán por la normalización de aquellos que integran la sociedad, siendo la organización, por excelencia, su medio para hacerlo (Foucault, 2001).

En este sentido, Foucault (1979) menciona que los mecanismos de poder no han sido muy estudiados en la historia, sino que han sido estudiadas las personas que detentan el poder. Una cosa que ha sido aún menos estudiada, es el conjunto de relaciones entre el poder y el saber, las incidencias de uno sobre el otro, pues existe una perpetua articulación del poder sobre el saber y del saber sobre el poder. Es decir, "el ejercicio del poder crea perpetuamente saber e inversamente el saber conlleva efectos de poder" (Foucault, 1979: 99).

Esta concepción del poder, no implica sin embargo una idea determinista y alienada de los sujetos. Donde hay poder hay resistencia al poder. Frente al poder o mejor dicho: intrínseco a él, se desarrollan resistencias múltiples y necesarias, improbables y posibles (Foucault, 2003). Esa resistencia se hizo manifiesta cuando diversos colectivos de profesores, principalmente de la Sección 22, del Sindicato Nacional de Trabajadores de la Educación (SNTE) de Oaxaca, o los también integrantes de la conocida Coordinadora Nacional de Trabajadores de la Educación (CNTE) -ala opositora a la agrupación oficialista-, con profesores de otros estados, como Guerrero, Michoacán y Chiapas, realizaron actividades de protesta tanto en sus entidades como en el Distrito Federal, llegando al extremo de bloquear carreteras; asalto a edificios que albergaban oficinas de educación y del Instituto Nacional Electoral, o con la obstrucción del acceso a aeropuertos y ocupación por periodos prolongados de espacios céntricos de diversas ciudades (Backhoff y Guevara, 2015).

Sin embargo, no debería asignarse un valor negativo a esa resistencia, pues su ejercicio contribuyó a la inclusión de puntos de vista que no habían sido considerados por el INEE. La resolución del conflicto, derivado de la relación poder-resistencia es desde, una perspectiva humana, el motor de una organización. En este sentido, será necesario establecer un debate sobre el análisis de las relaciones de poder y sobre las alternativas de participación (autonomía, colegialidad, etcétera) en la profesión docente.

Los profesores trabajan en un sistema educativo en el que se socializan personal y profesionalmente, ...su independencia profesional es, en todo caso, una aspiración, una conquista a obtener, no un punto de partida, esa realidad laboral de los profesores es inherente al papel que están llamados a cumplir en el sistema educativo (Gimeno, 1987).

Ante el reciente cambio de gobierno y con motivo de la entrada en vigor de la nueva reforma constitucional en materia educativa, publicada en el Diario Oficial de la Federación, el 15 de mayo de 2019, se derogan diversas disposiciones de los artículos $3^{\circ}, 31$ y 73 de la Constitución Política de los Estados Unidos Mexicanos; se ha declarado la cancelación de la reforma educativa de 2013 y la desaparición del INEE, así como la creación del Centro Nacional para la Revalorización del Magisterio y la Mejora Continua de la Educación, que tendrán como funciones, emitir lineamientos para elaborar programas de actualización y formación continua del magisterio; establecer y aplicar criterios con los que se verificarán los resultados de los programas de mejora y podrá presentar propuestas a las autoridades educativas, a fin de elevar la calidad de la educación (Poy y Muñoz, 2018).

Por lo tanto, la evaluación docente continúa, pues los maestros la aceptan, pero no ligada a lo laboral o la permanencia en el servicio, sino como una herramienta que permita conocer fallas e identificar áreas de oportunidad, para diseñar un modelo de formación para los docentes (Juárez, 2019). Desde esta perspectiva, puede notarse que la evaluación tiene múltiples finalidades, algunas de ellas, incluso contradictorias entre sí, al responder a distintos fines e intereses, cuyos efectos trascienden los límites de la escuela.

Aguerrondo y Xifra (2002) establecen diferenciaciones precisas entre una reforma y una novedad, pues ambas cambian aspectos fenoménicos: donde alguna cosa tiene que cambiar para que no cambie nada. Con la 
reforma, se transforman los elementos accesorios del sistema educativo, mientras que los cambios generados por la novedad impactan elementos superficiales a un nivel micro; como afirma Viñao (2002: 73): “...el relativo fracaso de las reformas educativas, se suceden una tras otra, arañando sólo superficialmente lo que sucede en los centros docentes y en las aulas, se debe, entre otras causas, a un presentismo ahistórico que ignora la existencia de unas culturas escolares formadas por regularidades y tradiciones que gobiernan la práctica y organización de la enseñanza y el aprendizaje, y que constituyen un producto histórico".

Con estas reflexiones, no se da por concluida la revisión de los discursos sobre evaluación del desempeño docente, sino más bien, constituyen la apertura para nuevas propuesta y posicionamientos en el campo temático abordado.

\section{Referencias Bibliográficas}

Aguerrondo, Inés \& Susana Xifra. (2002). La escuela del futuro I. Cómo piensan las escuelas que innovan. Buenos Aires: Ed. Papers Editores.

Alvarado, Macarena, Gustavo Cabezas, Denise Falck, \& María Elena Ortega. (2011). "La evaluación docente y sus instrumentos: discriminación del desempeño docente y asociación con los resultados de los estudiantes" en Informe MINEDUC/PNUD [en línea]. Chile: PNUD. Disponible en: http://www.cl.undp.org/content/chile/es/home/library/poverty/i nformes_de comision es/la-evaluacion-docente-y-susintrumentos-discriminacion-del-dese.html [23 de marzo de 2018

Arnaut, Alberto. (2015). "La Reforma Educativa: del gozo al pozo" en Colegio de México oficial [video]. México: Colegio de México. Disponible

https://www.youtube.com/watch?v=aG9qsww_w3k $\left[\begin{array}{ll}16 & \text { de }\end{array}\right.$ septiembre de 2017]

Banco Mundial. (2011). Aprendizaje para todos. Estrategia de educación 2020 del Grupo del Banco Mundial. Resumen Ejecutivo. Washington: Grupo del Banco Mundial.

Barber, Michael \& Mona Mourshed. (2007). Cómo hicieron los sistemas educativos con mayor desempeño del mundo para alcanzar sus objetivos. [Informe McKinsey]. Santiago de Chile: McKinsey \& Co.

Barrera, Iván \& Robert Myers, (2011). Estándares y evaluación docente en México: el estado del debate. Chile: PREAL.

Bruns, Bárbara \& Javier Luque. (2014). Profesores excelentes. Cómo mejorar el aprendizaje en América Latina y el Caribe. Washington: Grupo del Banco Mundial.

Catalán, Jorge \& Mauricio González. (2009). “Actitud hacia la evaluación del desempeño docente y su relación con la autoevaluación en profesores básicos de Copiapó, La Serena y Coquimbo" en PSYKHE [en línea]. Vol. 18, núm. 2, pp. 97112. Disponible en línea en: http://www.redalyc.org/articulo.oa?id=96711850007 [20 de enero de 2018]

Casassus, Juan. 2010. "Las reformas basadas en estándares: un camino equivocado" en Educere et Educare, Revista de Educação [en línea]. Vol. 5, núm. 9, pp. 85-107, enero-junio. Disponible en línea en: http://erevista.unioeste.br/index.php/educereeteducare/article/view/408 2 [18 de enero de 2017]

Cordero, Graciela \& Coral González. (2016). “Análisis del Modelo de Evaluación del Desempeño Docente en el Marco de la Reforma Educativa Mexicana" en Education Policy Analysis Archives/Archivos Analíticos de Políticas Educativas [en línea]. Vol. 24, pp. 1-26. Disponible en línea en: http://www.redalyc.org/articulo.oa?id=275043450025 [18 de junio de 2017]

Cuenca, Ricardo. (2015). Las carreras docentes en América Latina. La acción meritocrática para el desarrollo profesional. Estrategia Regional Sobre Docentes. Santiago de Chile: OREALC/UNESCO.

Diario Oficial de la Federación [DOF]. (2013). Ley General del Servicio Profesional Docente [en línea]. México: DOF. Disponible en línea en: http://www.diputados.gob.mx/LeyesBiblio/pdf/LGSPD.pdf [20 de septiembre de 2018]

Díaz-Barriga, Ángel. (2016). "Hacia una alternativa en Educación” en Educación Futura [en línea]. Disponible en línea en: http://www.educacionfutura.org/hacia-una-alternativa-angeldiaz-barriga/ [16 de diciembre de 2017]

Díaz-Barriga, Frida. (2010). "Los profesores ante las innovaciones curriculares" en Revista Iberoamericana de Educación Superior (RIES). Vol. I, núm. 1, pp. 3757. México: UNIVERSIA/IISUE

Echeverría, Rafael. 2003. Ontología del lenguaje. Santiago de Chile: J.C. Sáez Editor.

Fardella, Carla. (2012). "Verdades sobre la docencia, efectos y consecuencias subjetivas de la evaluación docente en Chile" en Revista de Psicología [en línea]. Vol. 21, núm. 1, pp. 209-227. Disponible en línea en: http://www.revistapsicologia.uchile.cl/index.php/RDP/article/vi ew/19996/0 [15 de febrero de 2017]

Feldman, Daniel, \& Gustavo F. Iaies. 2010. Competencias docentes: un marco conceptual para su definición [en línea]. Argentina: Fundación del Centro de Estudios en Políticas Públicas (CEPP). Disponible en línea en: https://vdocuments.site/amp/competencias-docentes-un-marcopara-su-definicion-preliminarpdf.html [18 de marzo de 2018]

Flores-Crespo, Pedro. (2016). “Dónde quedó la rectoría del Estado?” en Campus Milenio [en línea]. Disponible en línea en: http://campusmilenio.mx/index.php/template/opinion/universid adcritica/item/3845- donde-quedo-la-rectoria-del-estado [12 de mayo de 2017.]

Foucault, M. (1979). Microfísica del Poder. Madrid: Las Ediciones de la Piqueta.

Foucault, M. (1980). El ojo del poder (Vigésima sexta ed.). (J. Varela, \& F. Alvarez-Uria, Trads.) Barcelona: Las Ediciones de la Piqueta.

Foucault, M. (2001). Los anormales. Curso en el Collège de France (1974-1975) (2 da. ed.). (H. Pons, Trad.) México: FCE.

Foucault, M. (2003). Vigilar y Castigar. Buenos Aires: Siglo XXI Editores.

Fullan, Michael. (2002). Los nuevos significados del cambio en la Educación. Barcelona: Editorial Octaedro.

Galaz, Alberto. (2015). "Evaluación e identidad profesional del profesor: ¿Un juego de espejos rotos?” en Andamios [en línea]. 
Vol. 12, núm. 27, pp. 305-333. Disponible en línea en: http://www.scielo.org.mx/scielo.php?script=sci_arttext\&pid=S $187000632015000100015 \& \operatorname{lng}=e s \& \ln g=e s[13$ de marzo de 2017]

Gil Antón, Manuel. (2015). "Foro: Reforma educativa y evaluación docente: el debate" en IISUEUNAM oficial [video]. Minuto 27:27. Disponible en línea en: https:// www.youtube.com/watch?v=VYcyijkja64 [26 de abril de 2017]

Gil Antón, Manuel. (2016). "Usted perdone: estamos aprendiendo" en $E l$ Universal. Disponible en línea en: http://www.eluniversal.com.mx/entradadeopinion/articulo/manuelgilanton/nacion/2016/01/16/ustedperdone-estamos-aprendiendo [ 12 de enero de 2017]

INEE. (2015). Los docentes en México. Informe 2015 [en línea]. México: INEE. Disponible en línea en: http://publicaciones.inee.edu.mx/buscadorPub/P1/I/240/P1I240.pdf [16 de julio de 2018]

INEE. (2017). ¿Qué es el INEE? [en línea] México: INEE. Disponible en línea en: http://www.inee.edu.mx/index.php/acerca-delinee/que-es-el-inee [16 de julio de 2018]

Juárez, Erick. "Continuará evaluación docente pero no se ligará a lo laboral: Esteban Moctezuma" en Educación Futura [en línea]. Disponible en línea en: http://www.educacionfutura.org/continuara-evaluaciondocente-pero-no-se-ligara-a-lo-laboral-esteban-moctezuma/ [16 de febrero de 2019]

Poy, Laura \& Alma E. Muñoz. (2018). "Centro de revalorización del magisterio suplirá al instituto de evaluación educativa” en $L a$ Jornada [en línea]. Disponible en línea en: https://www.jornada.com.mx/2018/12/13/politica/007n1pol [16 de enero de 2019]

Leyva, Yolanda B., Sandra C. Serrato \& Marta Navarro (2015). "La buena enseñanza. Hacia la consolidación de un marco de la buena enseñanza en México. Perfil, parámetros e indicadores" en Revista RED [en línea]. Disponible en línea en: https://local.inee.edu.mx/images/stories/2015/revista red/2407/ Eje.pdf [12 de enero de 2018]

Manzi, Jorge. (2015). "Evaluación docente y desarrollo profesional: perspectivas y dilemas inspirados en la experiencia chilena" en Segundo Congreso Latinoamericano de Medición y Evaluación Educacional 2015 [en línea]. México: INEE. Disponible en línea

http://www.colmee.mx/index.php/colmee/conf/pages/view/pres entaciones [12 de febrero de 2018]

Manzi, Jorge, Roberto González, \& Yulan Sun. (2011). La evaluación docente en Chile. Santiago de Chile: Facultad de Ciencias Sociales, Escuela de Psicología-MIDE-UC.

Martínez-Chairez, Guadalupe I., Albertico Guevara \& María Manuela Valles. (2016). "El desempeño docente y la calidad educativa" en Ra Ximhai [en línea]. Vol. 12, julio-diciembre. Disponible en línea en: http://www.redalyc.org/articulo.oa?id=46148194007> ISSN 16650441 [29 de marzo de 2018]

Meckes, Lorena. (2014). "Estándares y formación docente inicial" en Temas críticos para formular nuevas políticas docentes en América Latina y el Caribe: El debate actual. Santiago de Chile: OREAL/UNESCO.

Moreno-Bayardo, M. Guadalupe. (2016). Culturas sobre la evaluación en los sistemas educativos. A propósito de la Reforma Educativa en México. Guadalajara: Universidad de Guadalajara.

Murillo, Francisco J., Verónica González \& Héctor H. Rizo. (2007). Evaluación del desempeño y carrera profesional docente. Una panorámica de América y Europa. Santiago de Chile: UNESCO.
Murillo, Francisco J. \& Marcela Román. (2010). "Retos en la evaluación de la calidad en América Latina" en Revista Iberoamericana de Educación [en línea], núm. 53, pp. 97-120. Disponible en línea en: http://www.rieoei.org/rie53a04.pdf [12 de mayo de 2017]

Navarro, Manuel. (2017). "Evaluar... ¿Para qué?” en Blog personal [en Línea]. Disponible en línea en: https://manuelnavarrow.com/2017/03/01/evaluar-para-que/ [16 de mayo de 2018]

OREALC/UNESCO. (2013). Antecedentes y Criterios para la Elaboración de Políticas Docentes en América Latina y el Caribe. Santiago de Chile: OREALC.

OCDE. (2010). "Acuerdo de cooperación México-OCDE para mejorar la calidad de la educación de las escuelas mexicanas" en Establecimiento de un marco para la evaluación e incentivos para docentes: Consideraciones para México [en línea]. Disponible en línea en: de http://www.oecd.org/education/school/46216786.pdf $\quad\left[\begin{array}{ll}12 & \text { de }\end{array}\right.$ agosto de 2019]

OCDE. (2010a). Specific Policy Recommendations on the Development of a Comprehensive In-Service Teacher Evaluation Framework [en línea]. Disponible en línea en: http://www.oecd.org/edu/school/48481142.pdf $\quad[20$ de noviembre de 2018]

OCDE. (2010b). Reflexiones finales del Seminario OCDE-Harvard para líderes en reformas educativas para fortalecer la profesionalización docente en México [en línea]. Disponible en línea en: https://www.oecd.org/edu/school/44780063.pdf [20 de noviembre de 2018]

Ornelas, Carlos. (2013). "Entrevista a Carlos Ornelas" en Revista Siempre [en línea]. Disponible en línea en http://www.siempre.com.mx/2013/09/si-seda-marchaatrasenreforma-educativa-pena-nieto-se-acaba/ [12 de mayo de 2018]

Ornelas, Carlos. (2016). "Política educativa: 2016, la disputa prosigue" en Periódico Excélsior [en línea]. Disponible en línea en http://www.excelsior.com.mx/opinion/carlosornelas/2016/01/06 /1067113 [16 de septiembre de 2018]

Rodríguez, Roberto. (2015). "Foro: Reforma educativa y evaluación docente: el debate" en IISUEUNAM oficial [video]. Minutos 31.00 - 32.00. Disponible en línea en: https:// www.youtube.com/watch?v=VYcyijkja64 [26 de abril de 2017]

Scott David, Charles Posner, Christopher Martin \& Elsa Guzmán. (2015). Intervention in Education Systems. London: Bloomsbury Academic.

SEP. (2010). Estándares de Desempeño Docente en el Aula para la Educación Básica. Borrador para Discusión [en línea]. México: SEP. Disponible en línea en: http://asignaturadeartes.files.wordpress.com/2011/11/estandadr es_desemp_doc_en

el_aula_de_educ_basica_en_mexico_esp.pdf [12 de septiembre de 2018]

SEP. (2015). Etapas, aspectos, métodos e instrumentos. Proceso de Evaluación del Desempeño docente. Educación Básica. Ciclo escolar. 2015-2016 [en línea]. Disponible en línea en: http://servicioprofesionaldocente.sep.gob.mx/content/ba/docs/2 015/permanencia/etapas aspectos/VERSION FINAL dmj docentes 190515.pdf $\quad[12$ de septiembre de 2018]

Sierra-Bravo, Restituto. (1999). Técnicas de investigación social, teoría y ejercicios $\left(13^{\mathrm{a}}\right.$ ed.). Madrid: Paraninfo.

Tornero, Bernardita \& Sandy Taut. (2010). "A mandatory, high-stakes National Teacher Evaluation System: Perceptions and attributions of teachers who actively refuse to participate" en Studies in Educational Evaluation. Vol. 36, Núm. 4, pp. 132142. 
Vaillant, Denise. (2004). Construcción de la profesión docente en América Latina. Tendencias, temas y debates [en línea]. PREAL. Disponible en línea en: http://www.oei.es/docentes/articulos/construccion_profesion_d ocente AL vaillant.pdf [21 de marzo de 2018].

Vázquez, María del Ángel; Graciela Cordero \& Yolanda Leyva. (2014). "Análisis comparativo de criterios de desempeño profesional para la enseñanza en cuatro países de América" en Revista Actualidades Investigativas en Educación [en línea]. Vol. 14, núm. 3, pp. 1-20. Disponible en línea en: http://www.redalyc.org/pdf/447/44732048018.pdf [15 de abril de 2018]

Vega Javier F. A., y Alberto Galaz. (2015). "Evidencias para una transformación y complejización del modelo chileno de evaluación docente" en Educação e Pesquisa [en línea]. Vol. 41, núm. 1, pp. 171-183. Disponible en línea en http://www.scielo.br/scielo.php?script=sci_arttext\&pid=S15179 7022015000100171\&lng=en\&nrm=iso\&tlng=es [15 de abril de 2018]

Viñao, Antonio. (2002). Sistemas educativos, culturas escolares y reformas. Madrid, España: Morata.

Zebadúa, Emilio. (2013). "Reforma educativa en México" en La crónica de hoy [en línea]. Disponible en línea en http://www.cronica.com.mx/ notas/2011/556315.html [28 de abril de 2018]

\section{NOTAS}

\footnotetext{
${ }^{\text {i }}$ PRI: Partido Revolucionario Institucional; PAN: Partido Acción Nacional; PRD: Partido de la Revolución Democrática.
} 